\title{
Regularization Rotational Motion Blur Image Restoration Based on Mixed Norm
}

\author{
Yunchao Luo ${ }^{\mathrm{a}}$, Huaping Zhu ${ }^{\mathrm{b}}$ and Yunyun Zhu ${ }^{\mathrm{c}}$ \\ School of Science, Wuhan University of Technology, Wuhan 430070, China \\ a913152031@qq.com, b65779221@qq.com, ‘469035934@qq.com
}

Keywords: rotational blur, rotational model, alternative separable function algorithm.

\begin{abstract}
Motion blur is very common in the blurred images, and rotating motion blur is one kind of special and general fuzzy form. Based on the principles of linear motion blur restoration, to change the rotary motion into linear motion blur, this paper propose a regularization recovery mode which is equipped with a norm 11-12, and with the help of alternative separable function algorithm to solve the model. As a result of the regularization method of norm 11-12to the noise and ringing has a good inhibitory effect is that the recovery effect is very good.
\end{abstract}

\section{Introduction}

Image restoration is important in image processing. The specified degradation model can rejuvenate degraded images. Rotary motion blurred image is working in the high speed rotating platform. To some extent, there is considerable relative rotation angle between Imaging system in a short exposure time and the target, which results in a blurring of the acquired picture.

Rotating motion blur is variable space, the farther from the image center of rotation, the lager fuzzy parameter is, and the higher the fuzzy degree is. The early researchers have provided many effective resolutions about the restoration of rotational motion blurred images. Sawchuk [1] puts forward a method for restoration based on geometric coordinate transformation. Hanyu Hong [2] presents the restoration of motion blur under the boundaries of non-zero values, and provide when fuzzy path beyond the pixel plane based on domain knowledge to guide the forehead secondary recovery algorithm. Shuyue Chen [3] puts forward the restoration of rotational motion blurred images based on difference method. First, the rotation of images should be change into linear motion blurring, and recover the image of transformation by difference method, then convert restoration image into the appropriate coordinate position.

First, this paper introduces the model of rotating motion blur, the degradation function of rotating motion blur is derived, and from the angle of solving linear system of equations, common least-squares filtering algorithm is described, to show the advantage of regularization method on image restoration, then presents restoration algorithm based on regularization strategy, to solve the model with the help of alternative separable function algorithm.

\section{The model of rotational motion blurred images}

Generally, the model of motion blur is

$$
g(x, y)=\int_{0}^{T} f\left(x-x_{0}(t), y-y_{0}(t)\right) d t
$$

$T$ is exposure time, $f(x, y)$ is the original image, $g(x, y)$ is blurry image, $x_{0}(t), y_{0}(t)$ is fuzzy path.

Though the pixel point is the line integral and superimposed along the fuzzy trajectory, in the process of the rotational motion blur, the fuzzy path is a series of concentric circles, and the model of rotational motion blurred images is

$$
g(x, y)=\int_{0}^{T} f(x-r \cos (\omega t), y-r \sin (\omega t)) d t
$$


We noted that $\omega$ is angular velocity, $r$ is the distances from the rotation center to the pixel point. We write the equation in a form that corresponds with the polar geometry

$g(l, r)=\frac{1}{T} \int_{0}^{T} f(r, l-s) d s$

Satisfying $l=r \omega T, s=r \omega t$

By one-dimensional equation:

$g_{r}(l)=\frac{1}{a_{r}} \int_{0}^{a_{r}} f_{r}(l-s) d s$

In order to carry on the type of discretization, we use Discrete coordinates i to present it:

$g_{r}(i)=\frac{1}{a_{r}}\left[f_{r}(i)+f_{r}(i-1)+\cdots+f_{r}\left(i-a_{r}+1\right)\right]$

We note that $a_{r}$ is blur extent, $h(x)$ is point spread function, we write it by the form of discrete convolution:

$g_{r}(i)=\sum_{m=0}^{N_{R}-1} f_{r}(m) h_{r}(i-m)=f_{r}(i) * h_{r}(i)$

Which is expressed in the matrix form, then get singular matrix:

$$
H=\left(\begin{array}{ccccc}
h(0) & h(N-1) & \cdots & h(2) & h(1) \\
h(1) & h(0) & \cdots & h(3) & h(2) \\
\vdots & \vdots & \vdots & \vdots & \vdots \\
\vdots & \vdots & \vdots & \vdots & \vdots \\
& & \vdots & \vdots & \vdots \\
h(N-2) & \cdots & h(1) & h(0) & h(N-1) \\
h(N-1) & \cdots & h(2) & h(1) & h(0)
\end{array}\right)
$$

\section{Image restoration algorithm}

Image restoration is a typical "pathological" problem. Pathological inverse problem can be directly solved by the least square algorithm, more classic method is least square method ${ }^{[4]}$, Its central idea is estimated data by minimizing the error between actual data and the square to search the optimal solution of the problem, $\hat{f}=\arg \min _{f}\|H f-g\|^{2}$. But the result is instability through this method, if there is a little disturbance about $g$, the result will be having great fluctuations. About this problem, the regularization method can effectively suppress the high frequency oscillation phenomena, so this paper use the $l_{1}-l_{2}$ norm regularization model to solve image restoration

$$
\hat{f}=\arg \min _{f}\left\{\frac{1}{2}\|H f-g\|_{2}^{2}+\lambda\|x\|_{1}\right\}
$$

In this model, $l_{1}=\lambda\|x\|_{1}$ represents the constraints, $l_{2}=\frac{1}{2}\|H f-g\|_{2}^{2}$ represents the fidelity term.

There are many methods to solve the mixed norm model, common methods include the original dual interior-point method and iterative-shrinkage algorithm .This article uses separable alternative method $^{[5]}$ which one of iterative-shrinkage algorithm.

Based on the approximate point algorithm of the optimization method ,the basic idea of the separable alternative method is upper boundary function of objective function $f(x)$ in the optimization problem. The separable alternative method referred to as "SSF algorithm", The method is to use separability of $l_{1}$ to get the analytic solution directly when minimizing the boundary function. 
$Q_{1}^{\lambda}: \min _{x} \operatorname{imize} f(x)=\frac{1}{2}\|A x-b\|_{2}^{2}+\lambda\|x\|_{1}$

$g(x)=\frac{1}{2}\|A x-b\|_{2}^{2}$

Using Taylor formula in $x=x_{0}$ about $g(x)$, it can be expressed as

$g(x)=g\left(x_{0}\right)+\nabla g\left(x_{0}\right)^{T}\left(x-x_{0}\right)+\left(x-x_{0}\right)^{T} A^{T} A\left(x-x_{0}\right)$

With $c I$ to replace the type of $A^{T} A, I$ is a unit matrix, $c$ as the constant, and $c \geq\left\|A^{T} A\right\|_{2}$. To define a new function

$\hat{g}(x)=g\left(x_{0}\right)+\nabla g\left(x_{0}\right)^{T}\left(x-x_{0}\right)+c\left\|x-x_{0}\right\|_{2}^{2}$

Obviously, $0 \leq g(x) \leq \hat{g}(x)$, so the objective function has the same properties

$$
f(x)=g(x)+\lambda\|x\|_{1} \leq \hat{f}(x)=\hat{g}(x)+\lambda\|x\|_{1}
$$

Therefore, $\hat{f}(x)$ can be regarded as upper boundary function of the objective function $f(x)$.

First of all , to solve the optimal solution of $\hat{f}(x)$, further

$$
\begin{aligned}
\hat{f}(x) & =g\left(x_{0}\right)+\nabla g\left(x_{0}\right)^{T}\left(x-x_{0}\right)+c\left\|x-x_{0}\right\|_{2}^{2}+\lambda\|x\|_{1} \\
& =\text { const }+\lambda\|x\|_{1}+c\|x-v\|_{2}^{2}
\end{aligned}
$$

Among the expression $v=x_{0}-\frac{1}{2} \nabla g\left(x_{0}\right)$

By separating elements of vector in the function $\hat{f}(x)$ and accumulate them, so the expression as

$$
\hat{f}(x)=\text { const }+\sum_{j=1}^{N}\left\{\lambda|x[j]|+c(x[j]-v[j])^{2}\right\}
$$

Among the expression, $x[j]$ and $v[j]$ respect first $j$ elements of vector $x$ and $v$, and $j=1,2, \cdots, N$.If we know $x_{0}$, we can get $v$, so we can get the minimum solution of $\hat{f}(x)$, Suppose this solution as $x_{1}$

$$
x_{1}[j]= \begin{cases}0 & |v[j]| \leq \frac{\lambda}{c} \\ v[j]-\frac{\lambda}{c} * \operatorname{sign}(v[j]) & \text { others }\end{cases}
$$

In this expression, $\operatorname{sign}(\bullet)$ as a symbol function, if $x \geq 0, \operatorname{sign}(x)=1$, and if $x<0, \operatorname{sign}(x)=-1$

In order to express simplely, the expression can be as

$$
x_{1}=S_{\lambda / c}(v)=S_{\lambda / c}\left(\frac{1}{C} A^{T}\left(b-A x_{0}\right)+x_{0}\right)
$$

$S$ is a contraction function and $\lambda / c$ is a contraction threshold.

The process of SSF algorithm as follow

The initial value $x_{0}$, the initial residual value $r_{0}=b-A x_{0}$, constant $c=1.01 *\left\|A^{\prime} A\right\|_{2}$

The iteration: the first $\mathrm{k}$ iteration, do the next step

Calculate: $e=A^{\prime} r_{k-1}$

Calculate: $e_{s}=S_{\lambda / c}\left(x_{k-1}+e / c\right)$

a. Linear search: Along the direction down to find the best step length

Update the solution: $x_{k}=x_{k-1}+\mu\left(e_{s}-x_{k-1}\right)$

Update the residual: $r_{k}=b-A x_{k}$

Stop condition: If $\left\|x_{k}-x_{k-1}\right\|_{2} \leq$ somenumber, Stop the iteration ;Otherwise enter the $k+1$ iteration

The output: After $k+1$ iteration convergence and solution as $x_{k}$ 
Rotating motion blur is variable fuzzy space, if we implement the above algorithms directly, we can't restore the rotate blurred image, so we will convert rotate the fuzzy problem into linear fuzzy to recover. Assumes that the center of rotation as $O$, The distance of radius from the center of the circle as $r$.According to the $r$ raised to divide the image into different sizes of concentric circles, by the Bresenham Method, we extract all the pixels on the concentric circles according to the direction of rotation along the fuzzy path. Next we take them as row vector $g_{1}, g_{2}, \cdots, g_{n}$, so the rotating motion blur can approximate linear motion blur.

\section{Image restoration result}

In the experiment, the size of the rotary motion blurred image as $231 \times 231$, the image center as center of rotation, and the angle of rotation is $30^{\circ}$. Recovery effect as shown in figure:

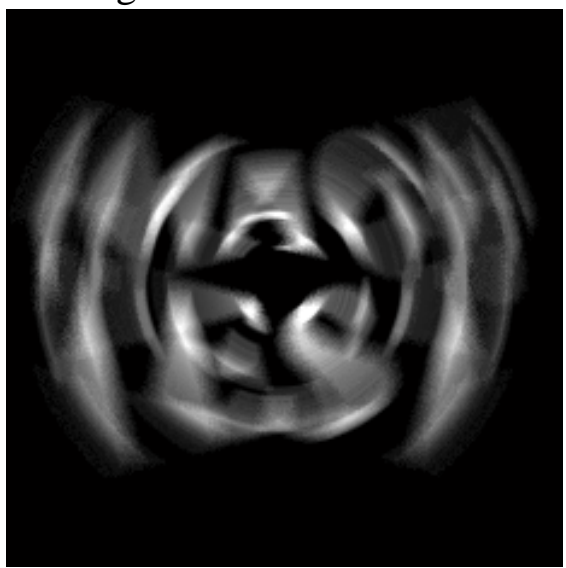

Fig. 1 Rotating motion blur

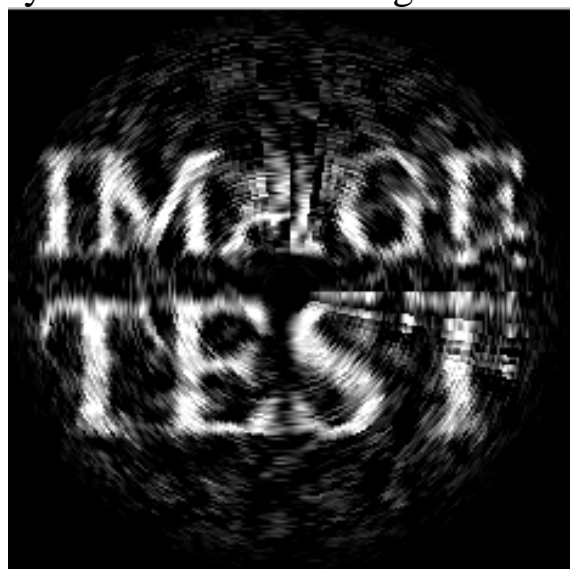

Fig. 2 Recovery clear figure

\section{Conclusion}

In this paper, the image is divided into many concentric circles based on the center of the circle center of rotation .And based on the least squares algorithm, we replace $l_{2}$ norm with $l_{1}$ norm, and using SSF algorithm to solve the $l_{1}-l_{2}$ mixed norm of the linear problem. This algorithm is not sensitive to outliers, and can effectively handle noise factors.

\section{Acknowledgements}

This work was supported by the Fundamental Research Funds for the Central Universities under Grant 2013-la-033

\section{References}

[1] Sawchuk A.A..Space variant image restoration by corrdinate transformations.J.Opt.Soc.Am. A, 1974, 64(2):138-144.

[2] HONG Han-yu, ZHANG Tian-xu. Restoration Algorithm for Rotational Motion Blurred Images with Non-zero Boundary. In Journal of Images and Graphics, 2004.

[3] CHEN Shu-yue, FENG Jun , ZOU Ling. Study of Image Restoration under Rotation Motion Blurring Based on Spatial Difference Technique.In Microelectronics\&Computer,2011.

[4] Bjorck A. Numerical methods for least squares problems.Philadelphia: Society for Industrial and Appiled Mathematics (SIAM), 1996.

[5] Zibulevsky,M.and M.Elad, L1-L2 Optimization in Signal and Image Processing. IEEE Signal. Process.Mag, 2010.27:p.76-88. 